\title{
Screen-printed Flexible and Transparent Antenna
}

\author{
Weiwei Li, Azat Meredov, Kirill Klionovski, Atif Shamim \\ IMPACT Lab, Division of Computer, Electrical, and Mathematical Sciences and Engineering \\ King Abdullah University of Science and Technology (KAUST) \\ Thuwal 23955-6900, Saudi Arabia \\ weiwei.li@kaust.edu.sa,azat.meredov@kaust.edu.sa,kirill.klionovski@kaust.edu.sa, atif.shamim@kaust.edu.sa
}

\begin{abstract}
Optically transparent antennas have attracted considerable attention in the past few decades due to potential applications in solar panels and wireless communications. However, the designs are typically based on transparent conductive oxides, which suffer from low transparency, high cost and poor flexibility. In this paper, an optically transparent, mechanically flexible monopole antenna is presented, which has been realized through screen-printing of silver nanowires (Ag NWs) in high efficiency. The proposed monopole antenna has been printed on a $50-\mu \mathrm{m}$-thick polyethylene terephthalate substrate. The total size of the antenna is $12 \times 12 \mathrm{~mm}^{2}$, and the operating frequency covers $\mathrm{Ku}$ - and $\mathrm{Ka}-\mathrm{band}(18-40 \mathrm{GHz})$, which makes the antenna suitable for mm-wave applications including 5G communication. The antenna demonstrates excellent stability over 100 bending cycles with a radius of $4 \mathrm{~mm}$. More importantly, 89\% transmittance has been achieved at a wavelength of $550 \mathrm{~nm}$, which is better than transparent antennas in literature.
\end{abstract}

Keywords - transparent antenna, wideband, silver nanowire, flexibility.

\section{INTRODUCTION}

With the rapid development of the Internet of Things (IoT), electronic devices based on wireless communication has become a hot topic [1]. As an essential component of wireless communication, the antenna has drawn more and more attention. The common antennas are typically made of pure metals, which are opaque to visible light, enabling non-transparent visual to our eyes. Alternately, the optically transparent antennas have attracted growing interest due to the potential applications in solar panels, satellites, smart windows and other related areas. Typically, the transparent materials used in the fabrication of transparent antennas includes metal mesh and transparent conductive oxides, which have been extensively studied during the past few decades $[2,3]$. The fabricated transparent antennas can show more than $80 \%$ transparency with the design of various slotted structures. However, the transparent antennas made of these conductive materials shows no flexibility [4] due to the brittleness of the materials.

In this paper, a flexible, transparent, wideband monopole antenna has been designed, simulated, fabricated and tested. The conductive parts of the proposed antenna are screen-printed with Ag NWs, which is highly transparent and mechanically flexible. The working frequency of the antenna is from 15.5 to $45 \mathrm{GHz}$, making it suitable for $5 \mathrm{G}$ applications. The return loss shows no obvious change even after 100 bending cycles with a radius of $4 \mathrm{~mm}$.

\section{ANTENNA DESIGN AND FABRICATION}

\section{A. Antenna Design}

The design of the proposed transparent antenna is a triangular monopole antenna, which consists of a conductive triangle patch and a ground layer separated by a polyethylene terephthalate with dielectric constant of 3 and thickness of 0.05 $\mathrm{mm}$. The conductivity of the patch layer and ground layer is $10^{6}$ $\mathrm{S} / \mathrm{m}$. The structure and geometric parameters of the designed antenna are shown in Fig. 1. The simulated reflection coefficient (S11) of the antenna is depicted in Fig. 2, and it can be observed that the $-10 \mathrm{~dB}$ bandwidth of the antenna is from 15.5 GHz to $45 \mathrm{GHz}$, indicating ultra-wide bandwidth. The simulated maximum antenna gain is $0.8 \mathrm{dBi}$ at $30 \mathrm{GHz}$ as shown in Fig. 3. The radiation pattern is shown in Fig. 4.

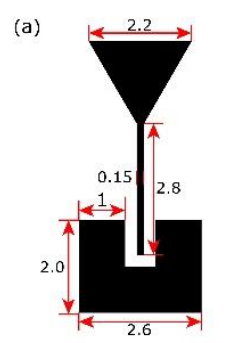

(b)
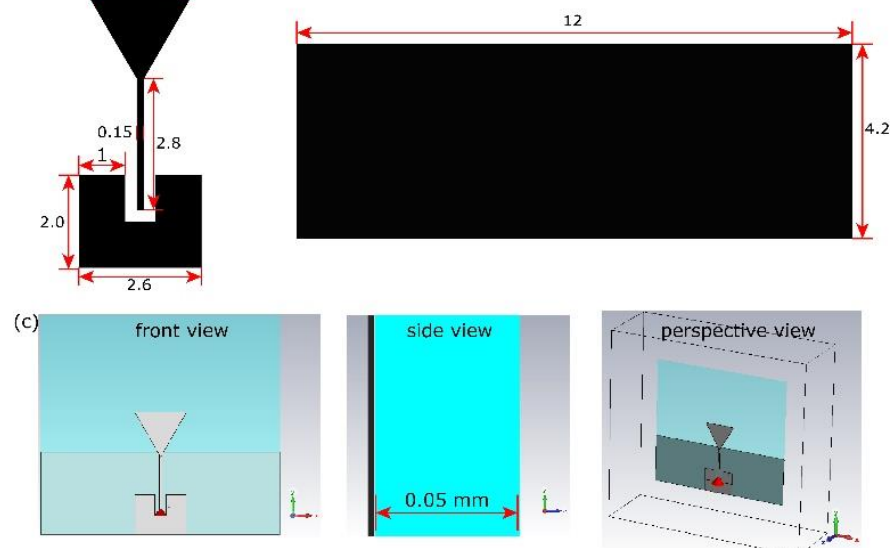

Fig. 1. Geometrical parameters and 3D model of the antenna. Unit is in $\mathrm{mm}$.

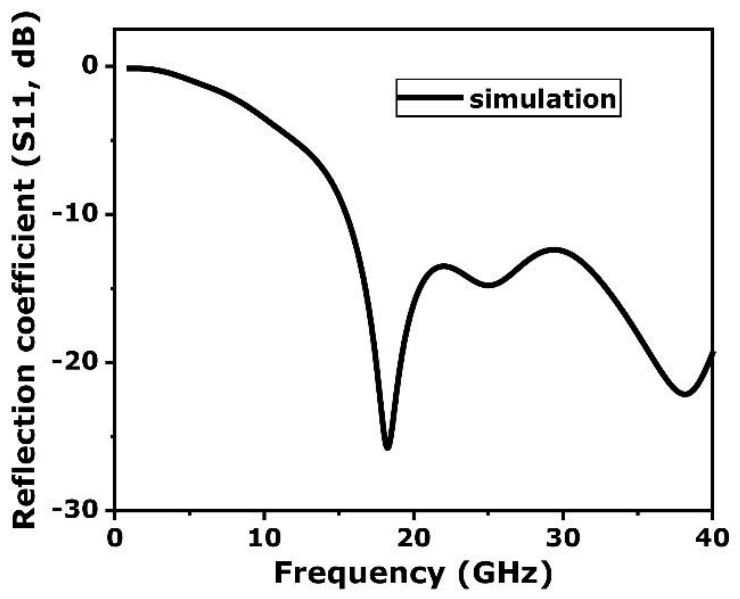

Fig. 2. Simulated reflection coefficient of the antenna. 


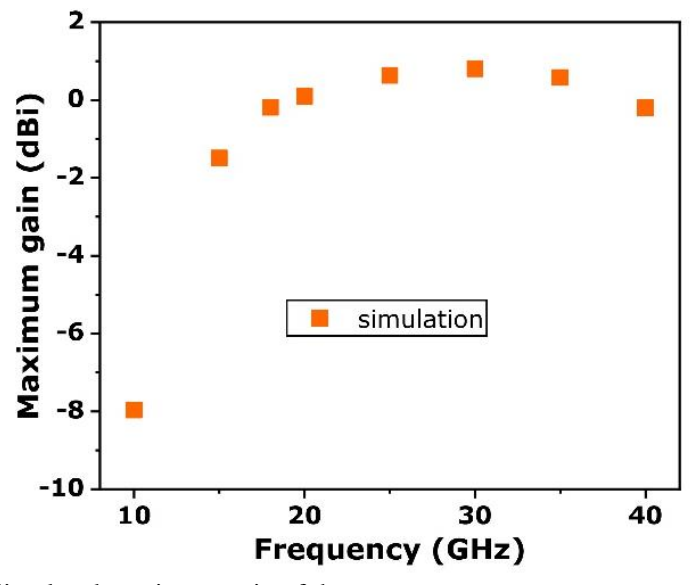

Fig. 3. Simulated maximum gain of the antenna.

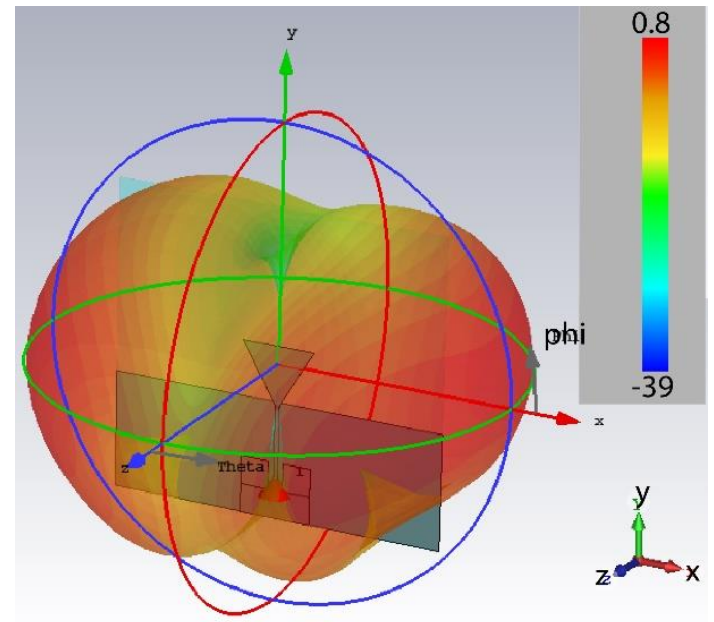

Fig. 4. Simulated radiation pattern of the antenna at $30 \mathrm{GHz}$.

\section{B. Antenna Fabrication}

The designed antenna has been fabricated through a screenprinting technique. Briefly, the substrate is firstly cleaned with water, acetone, and ethanol, followed by UVO plasma treatment for $2 \mathrm{~min}$. Then, $\mathrm{Ag} \mathrm{NW}$ ink was deposited onto the substrate by screen-printing. After completely dried in an oven at $80{ }^{\circ} \mathrm{C}$, the antenna was obtained. Inset of Fig. 6 shows six antennas printed in one cycle, indicating high efficiency of the fabrication process.

\section{RESULTS AND DISCUSSIONS}

\section{A. Reflection Coefficient}

A RF probe connected to a vector network analyzer (Agilent N5225A) was used to record S11 of the fabricated antenna (Fig. 5a). As shown in Fig. 5b, the measured and simulated S11 of the proposed antenna matched well with each other. The slight displacement between the simulation and measurement could be attributed to the misalighment of the top and bottom layer, and the ideal simulation model. To investigate the flexibility of the antenna, multicycle bending has been performed to the antenna. The S11 of the antenna shows no noticeable change after 100 bending cycles as compared with the as-prepared antenna (Fig. 5b). Despite the minor difference among the measured S11 after bending, the fabricated antenna demonstrates excellent flexibility and stability under mechanical deformation.

(a)

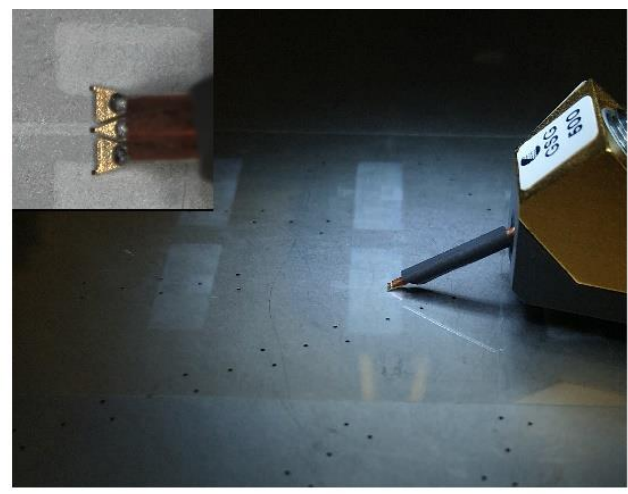

(b)

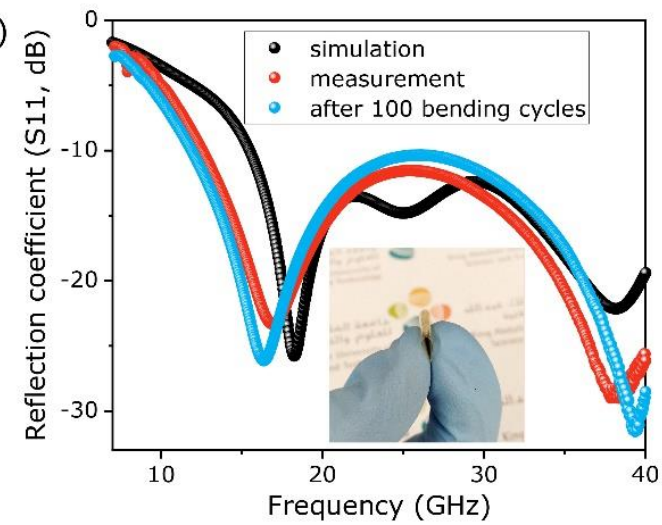

Fig. 5. (a) Digital photo of a setup to measure $\mathrm{S} 11$ of the antenna. Inset is a microscope image of the antenna under test. (b) Measured S11. Inset is an antenna under bending condition.

\section{B. Transparency of the Antenna}

The transparency of the fabricated monopole antenna has been investigated using a UV-Vis spectrophotometer by placing the antenna between two optical lenses. The optical transmittance was measured in the wavelength of $380-800 \mathrm{~nm}$, covering the entire visible light range. As shown in Fig. 6, it can be observed that more than $80 \%$ of transmittance for the antenna has been obtained and the transmittance at a wavelength of $550 \mathrm{~nm}$ is $89 \%$, which is better than micro-metal mesh antenna [5] and graphene antenna [6].

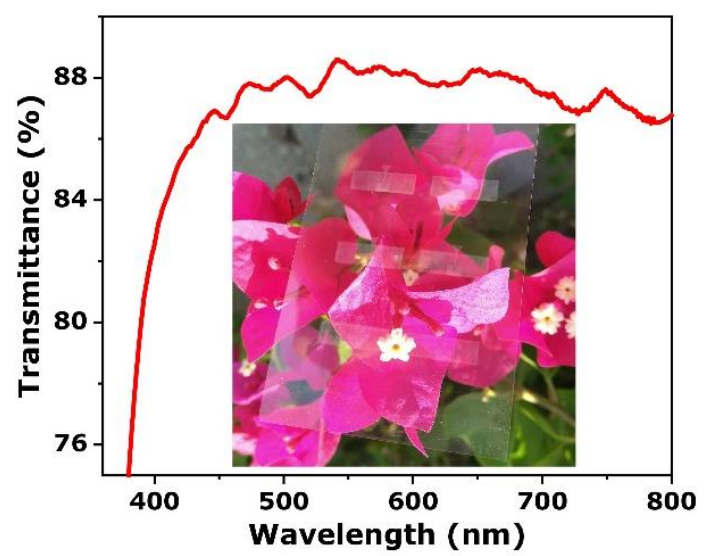

Fig. 6. The measured transmittance spectra of the fabricated antenna. Inset is digital photograph of the antenna. 


\section{CONCLUSION}

In conclusion, transparent and flexible antenna showing ultra-wide bandwidth for $5 \mathrm{G}$ applications has been designed, fabricated and measured. The measured S11 of the fabricated antenna agrees well with the simulated result. The antenna demonstrates excellent flexibility and stability under multicycle bending test. More than $80 \%$ transmittance has be achieved in the entire visible spectrum.

\section{REFERENCES}

[1] A. Sarycheva, A. Polemi, Y. Liu, K. Dandekar, B. Anasori, Y. Gogotsi, "2D titanium carbide (MXene) for wireless communication," Sci. Adv., vol. 4, pp. eaau0920, Sep. 2018.

[2] Y. Yao, W. Chen, X. Chen, and J. Yu, "Design of Optically Transparent Antenna with Directional Radiation Patterns," Int. J. Antennas Propag., vol. 2017, pp. 8125432, Aug. 2017.

[3] N. I. M. Ali, N. Misran, M. F. Mansor and M. F. Jamlos, "Transparent solar antenna of $28 \mathrm{GHz}$ using transparent conductive oxides (TCO) thin film," J. of Physics.: Conf. Series vol. 852, pp. 012036. May, 2017.

[4] G. Sun, B. Muneer and Q. Zhu, "A study of microstrip antenna made of transparent ITO films," 2014 IEEE Antennas and Propagation Society International Symposium (APSURSI), pp. 1867-1868, July, 2014.

[5] Li, Q. L.; Cheung, S. W.; Wu, D.; Yuk, T. I., "Optically Transparent Dual-Band MIMO Antenna Using Micro-Metal Mesh Conductive Film for WLAN System", IEEE Antennas Wirel. Propag. Lett, vol. 16, pp. 920-923, 2017.

[6] Grande, M.; Bianco, G. V.; Laneve, D.; Capezzuto, P.; Petruzzelli, V.; Scalora, M.; Prudenzano, F.; Bruno, G.; D'Orazio, A., "Optically transparent wideband CVD graphene-based microwave antennas", Appl. Phys. Lett. vol. 112, pp. 251103, 2018. 